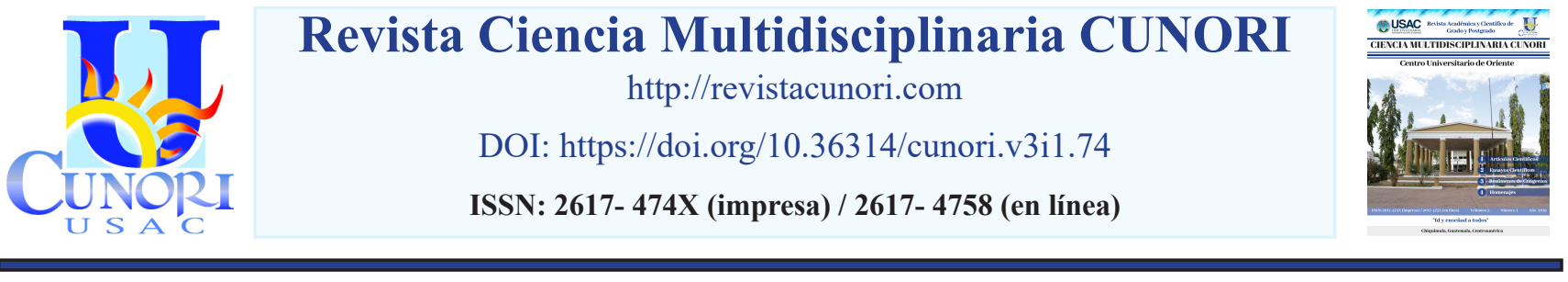

Como citar el artículo

Mariscal, P., Mazariegos, E., Farrington, J. (2019). Complicaciones maternas neonatales en pacientes preeclámpticas. Revista Ciencia Multidisciplinaria CUNORI, 3(1), 1-10. DOI: https://doi.org/10.36314/cunori.v3i1.74

\title{
Complicaciones maternas neonatales en pacientes preeclámpticas
}

\section{Maternal-neonatal complications in patients with preeclampsia}

\author{
Paola Mariscal*, Edvin Mazariegos, Jack Farrington \\ Centro Universitario de Oriente (CUNORI), Universidad de San Carlos de Guatemala
}

Recibido: 18 de julio de 2018 / Revisión: 08 de agosto de 2018 / Aceptado: 30 de septiembre de 2018

Disponible en internet el 30 de agosto de 2019

\author{
*Autor para correspondencia. \\ Resumen \\ Correo electrónico: pao.tatiana@hotmail.com
}

Preeclampsia es un síndrome específico del embarazo que puede afectar a todos los sistemas orgánicos, se define como el
estado hipertensivo acompañado de proteinuria el cual puede llevar a serias complicaciones e incluso a la muerte tanto de
la madre como del feto o neonato. En países en vías de desarrollo, una mujer tiene siete veces más probabilidades de desarrollar
preeclampsia, comparado con una mujer que vive en un país desarrollado. Se realizó un estudio descriptivo retrospectivo de 196
pacientes preeclámpticas y sus complicaciones maternas neonatales atendidas en el Hospital Regional de Zacapa, Guatemala, en
los años 2013 a 2017 . De las 196 pacientes preeclámpticas el $44 \%$ presentó complicaciones, siendo las más frecuentes el parto pre-
término con $37 \%$, el síndrome de Hellp con el $30 \%$, eclampsia $10 \%$ y oligohidramnios $10 \%$. No se presentó ningún caso de muerte
materna. De los 196 hijos de madres preeclámpticas el $61 \%$ presentaron complicaciones, las más frecuentes fueron bajo peso al
nacer con $21.5 \%$, pequeño para edad gestacional $20.6 \%$, restricción del crecimiento intrauterino con $20.3 \%$ y prematurez $12.9 \%$.

Palabras clave: preeclampsia, eclampsia, bajo peso al nacer, restricción del crecimiento intrauterino, prematurez

Abstract

Dreeclampsia is a specific syndrome of pregnancy that can affect all organic systems, it is defined as hypertensive state accom1 panied by proteinuria which can lead to serious complications and even the death of both the mother and the fetus or neonate. In developing countries, a woman is seven times more likely to develop preeclampsia than a woman who lives in a developed country. A retrospective descriptive study of 196 patients with preeclampsia and their maternal-neonatal complications attended at the Regional Hospital in Zacapa in the years 2013 to 2017 was conducted. Of the 196 patients with preeclampsia 44\% presented complications, being the most frequent preterm delivery 37\%, HELLP syndrome $30 \%$, eclampsia $10 \%$ and oligohydramnios $10 \%$. No case of maternal death was presented. Of the 196 children of mothers with preeclampsia $61 \%$ presented complications, the most frequent were low birth weight $21.5 \%$, small for gestational age $20.6 \%$, intrauterine growth restriction $20.3 \%$ and prematurity $12.9 \%$.

Keywords: preeclampsia, eclampsia, low birthweight, intrauterine growth restriction, prematurit 


\section{Introducción}

Preeclampsia se describe como un síndrome específico del embarazo que puede afectar a todos los sistemas orgánicos. Aunque la preeclampsia es mucho más que una simple hipertensión gestacional con proteinuria, la aparición de esta última se mantiene como un criterio diagnóstico objetivo importante. La proteinuria se define como la proteína en orina de $24 \mathrm{~h}$ mayor de $300 \mathrm{mg} / 24 \mathrm{~h}$, la existencia de un índice urinario proteína: creatinina $\geq 0.3$, o una concentración persistente de $30 \mathrm{mg} / \mathrm{dl}$ (1+ en pruebas con tira reactiva) de proteína en muestras aleatorias de orina según (Cunningham et al.,2015:728-730). En América Latina, la preeclampsia es la causa número uno de la muerte materna. En países en vías de desarrollo, una mujer tiene siete veces más probabilidades de desarrollar preeclampsia que una mujer que vive en un país desarrollado. De estos casos, entre el 10 y $25 \%$ de los casos terminarán en muerte materna, como lo afirma (Preeclampsia Foundation, 2014:1).

Los trastornos hipertensivos del embarazo son causa importante de morbilidad grave, discapacidad crónica y muerte entre las madres, los fetos y los recién nacidos. Entre los trastornos hipertensivos la preeclampsia sobresale por su impacto en la salud materna y neonatal. Es una de las causas principales de morbilidad y mortalidad materna y perinatal en todo el mundo. Según la (OMS 2014: 4), en América Latina, una cuarta parte de las muertes maternas se relacionan con esas complicaciones. El estudio tiene como objetivo describir las complicaciones maternas neonatales en pacientes preeclámpticas ingresadas en servicio de ginecología y obstetricia del Hospital Regional de Zacapa en el periodo de 2013 a 2017.

\section{Materiales y métodos}

Se realizó un estudio descriptivo retrospectivo sobre las complicaciones maternas neonatales en pacientes preeclámpticas ingresadas en el servicio de ginecología y obstetricia del Hospital Regional de Zacapa, Guatemala, en el periodo de 2013 a 2017. Se incluyeron 196 expedientes clínicos de pacientes con diagnóstico de preeclampsia y sus recién nacidos en el periodo establecido, fueron excluidas las pacientes con enfermedades concomitantes y cuyos parto no fueron atendidas en dicho hospital.

Las variables estudiadas fueron preeclampsia, complicaciones maternas (eclampsia, síndrome de Helpp, parto pretérmino, desprendimiento de placenta, hemorragia anormal posparto, coagulación intravascular diseminada, insuficiencia renal aguda, edema de pulmón, trastornos mentales, ingreso a unidad de cuidados intensivos, muerte) y complicaciones neonatales (prematurez, bajo peso al nacer, pequeño para edad gestacional, restricción del crecimiento intrauterino, ingreso a unidad de cuidados intensivos, muerte). Para la recolección de datos se revisaron los expedientes clínicos de todos los pacientes que cumplen los criterios de inclusión durante el período establecido, esta información se registró en una boleta de recolección de datos por número de registro hospitalario de cada paciente, los datos recolectados fueron tabulados y expresados gráficamente. Para la recolección de datos se revisaron los expedientes clínicos de todos los pacientes que cumplen los criterios de inclusión durante el período establecido, esta información se registró en una boleta de recolección de datos por número de registro hospitalario de cada paciente, los datos recolectados fueron tabulados y expresados gráficamente. 


\section{Resultados}

\section{Edad de las pacientes con diagnóstico de preeclampsia}

La edad que se observa con mayor frecuencia en las pacientes con diagnóstico de preeclampsia es la comprendida de 21-30 años representando el 44\% (87 casos), seguido de 15-20 años con 38\% (75 casos). Con menos frecuencia se encuentra $41-50$ años y $<15$ años con $2 \%$ ( 3 casos) y $1 \%$ ( 2 casos) respectivamente.

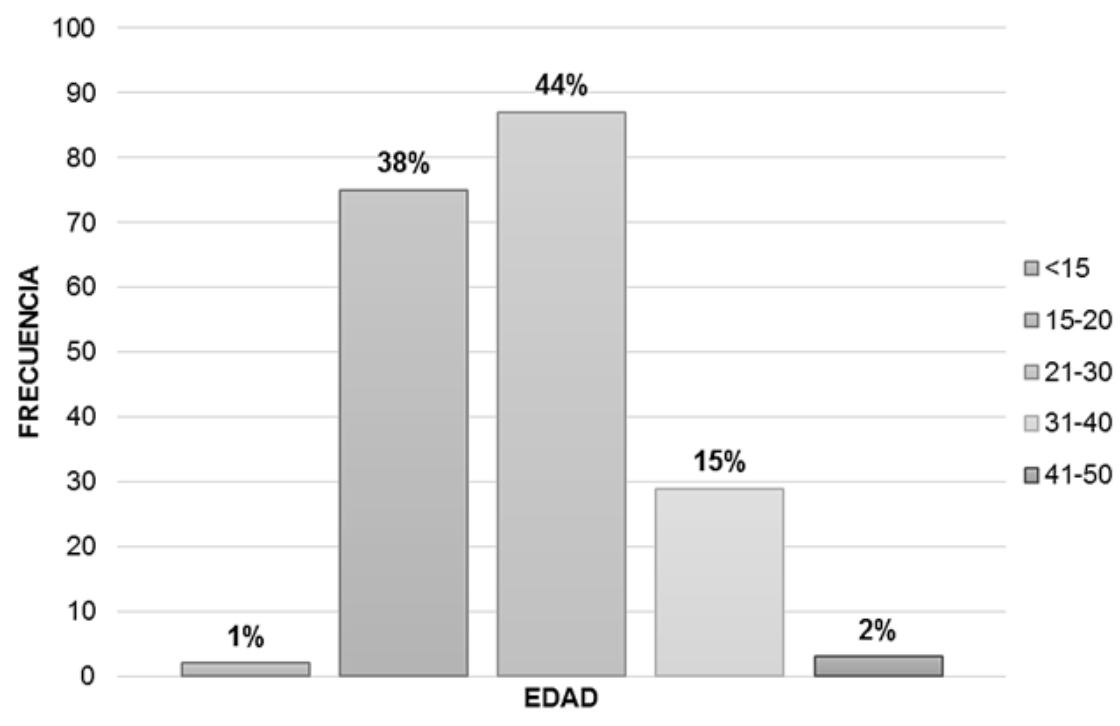

Figura 1. Distribución del grupo etario de pacientes con diagnóstico de preeclampsia atendidas en el Hospital Regional de Zacapa en el período del año 2013 al año 2017.

\section{Presencia de complicaciones maternas}

De las 196 pacientes preeclámpticas un 56\% (110 casos) no presentaron ninguna complicación, mientras que un $44 \%$ ( 86 casos) si presentaron algún tipo de complicación.

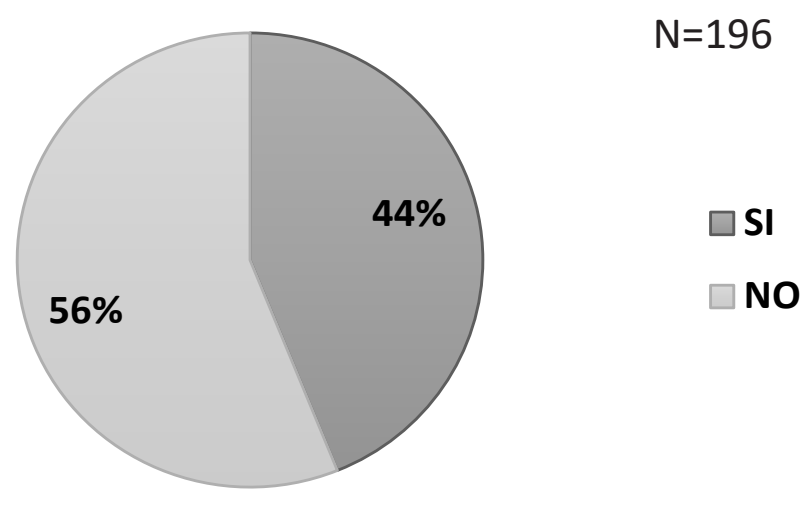

Figura 2. Distribución de pacientes preeclámpticas según presencia de complicaciones maternas atendidas en el Hospital Regional de Zacapa en el período del año 2013 al año 2017. 


\section{Tipo de complicación materna}

De las 196 pacientes preeclápticas la complicación materna más frecuente fue el parto pretérmino con 37\% (45 casos), seguido de síndrome de Hellp 30\% (36 casos), eclampsia 10\% (12 casos) y oligohidramnios $10 \%$ (12 casos). En menor frecuencia se encuentran hemorragia anormal posparto y retención de restos placentarios 4\% (5 casos) y 2\% (3 casos) respectivamente, seguido de anhidramnios y trastornos mentales ambos con $2 \%$ (2 casos) y por último placenta acreta, tromboembolia pulmonar, insuficiencia renal aguda, hematoma bilateral de la pared abdominal y edema de pulmón con 1\% (1 caso) cada uno.

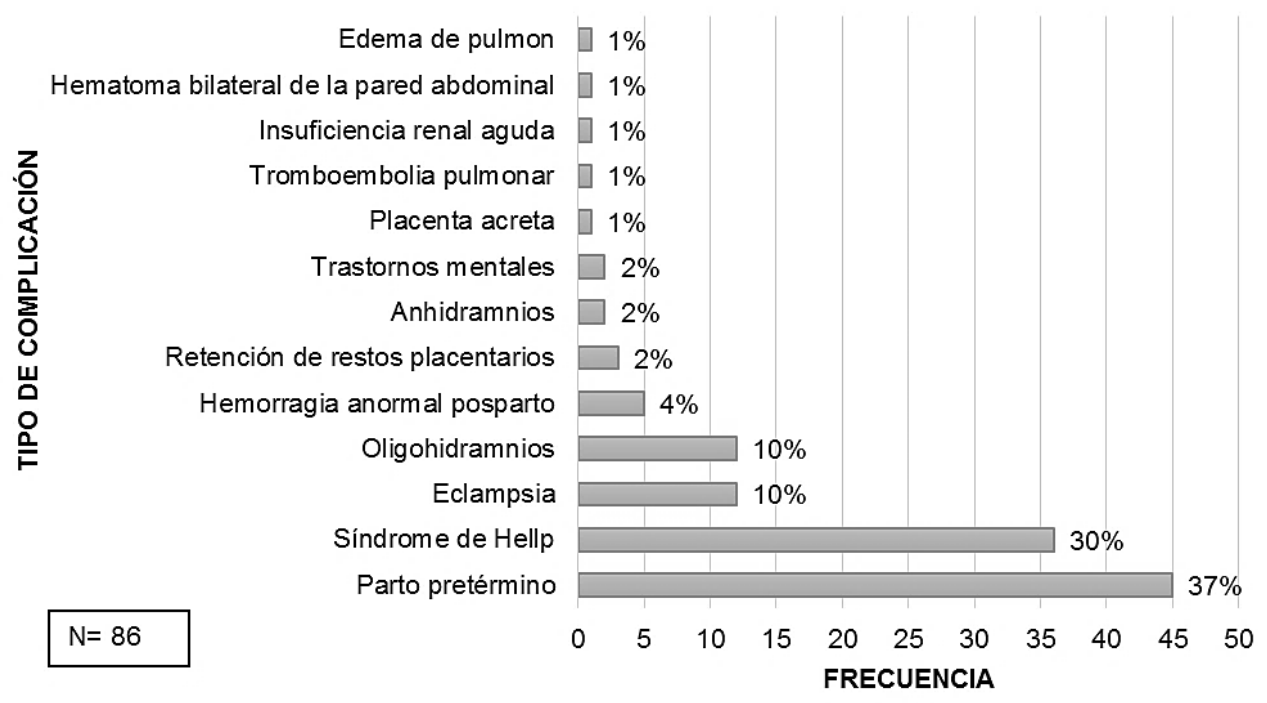

Figura 3. Distribución de pacientes preeclámpticas según tipo de complicación atendidas en el Hospital Regional de Zacapa en el periodo del año 2013 al año 2017.

\section{Sexo de hijos vivos de madres preeclámpticas}

De los 196 pacientes estudiados 52\% (102 casos) son de sexo masculino y 48\% (94 casos) son de sexo femenino, mostrando poca diferencia entre ambos.

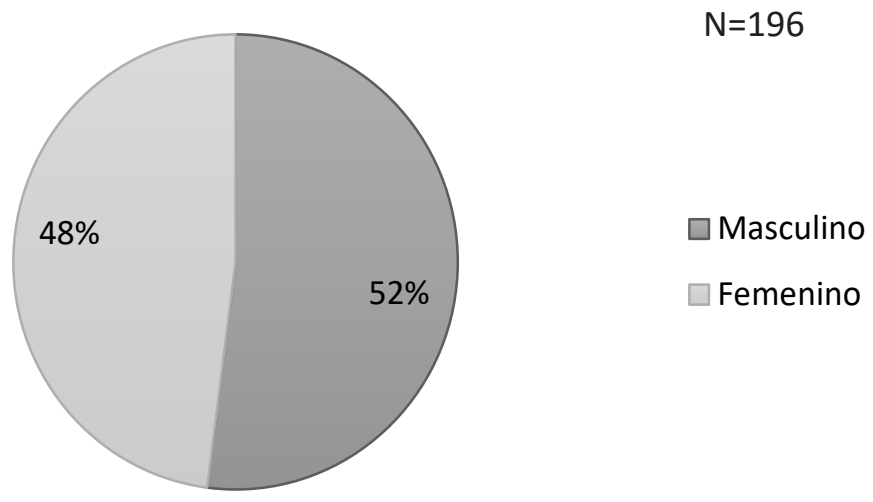

Figura 4. Distribución de pacientes hijos de madres preeclámpticas según la clasificación de bajo peso al nacer atendidos en el Hospital Regional de Zacapa en el período del año 2013 al año 2017. 


\section{Presencia de complicaciones en hijos de madres preeclámpticas}

De los 196 pacientes estudiados un 61\% (120 casos) de los pacientes estudiados presentaron algún tipo de complicación, mientras que un 39\% (76 casos) no presentó ninguna complicación.

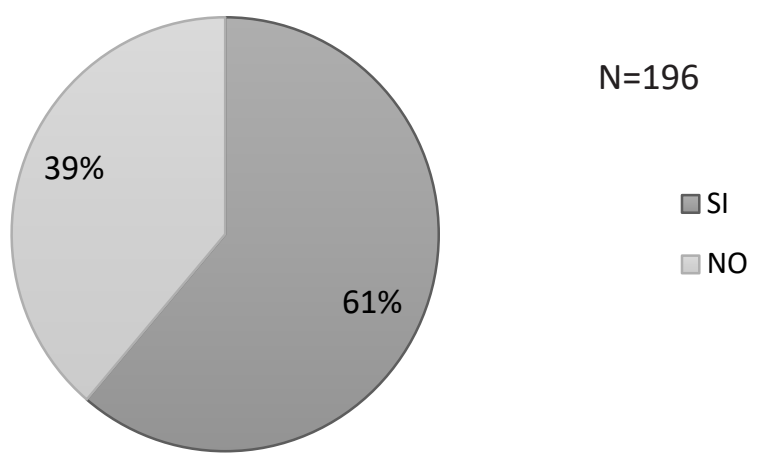

Figura 5. Distribución de pacientes hijos de madres preeclámpticas según presencia de complicaciones atendidos en el Hospital Regional de Zacapa en el período del año 2013 al año 2017.

\section{Tipo de complicación en hijos de madres preeclámpticas}

La complicación más frecuente de los recién nacidos hijos de madres preeclámpticas fue BPN (bajo peso al nacer) con 21.5\% (75 casos), seguido de PEG (pequeño para edad gestacional) con 20.6\% (72 casos), RCIU (restricción del crecimiento intrauterino) con $20.3 \%$ (71 casos) y prematurez con $12.9 \%$ 45 casos. Seguido de muerte 4.9\% (17 casos), SDR (síndrome de dificultad respiratoria) 4.3\% (15 casos), ingreso a UCIP (Unidad de Cuidados Intensivos Pediátricos) 4\% (14 casos), óbito fetal 2.9\% (10 casos), SAM (síndrome de aspiración meconial) y EMH (enfermedad de membrana hialina) 2\% (7 casos), APGAR bajo a los 5 min 1.7\% (6 casos). Por ultimo las complicaciones menos frecuentes fueron APN (asfixia perinatal), choque séptico, hipoglicemia, neumonía neonatal con $0.6 \%$ cada uno ( 2 casos) e ictericia $0.3 \%$ ( 1 caso).

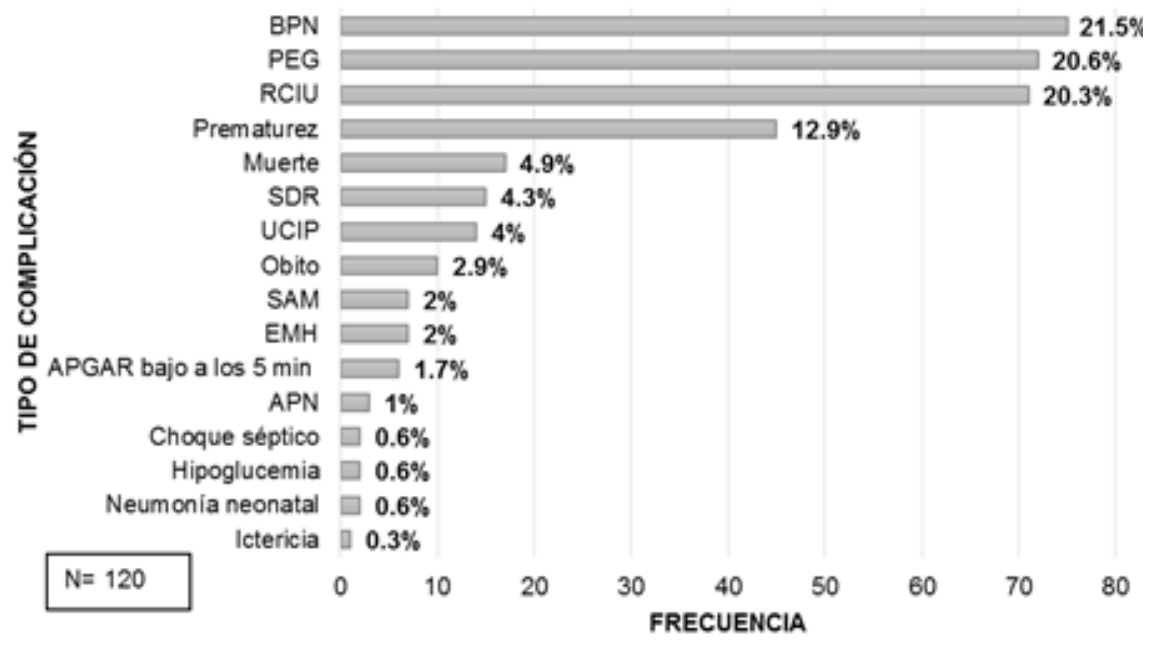

Figura 6. Distribución de pacientes hijos de madres preeclámpticas según tipo de complicación presentada atendidos en el Hospital Regional de Zacapa en el período del año 2013 al año 2017. 


\section{Clasificación de restricción del crecimiento intrauterino en hijos de madres preeclámpticas}

El 75\% (54 casos) de recién nacidos presentaron una restricción de crecimiento intrauterino tipo II, mientras que un $25 \%$ (18 casos) presentaron restricción de crecimiento intrauterino tipo I.

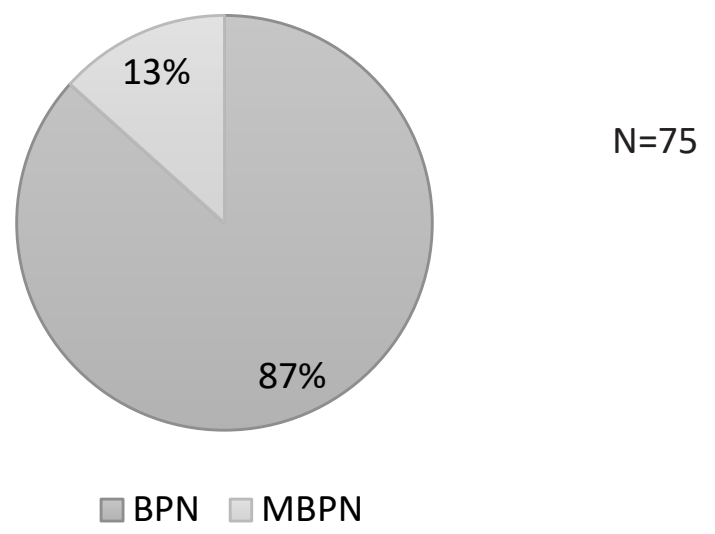

Figura 7. Distribución de pacientes hijos de madres preeclámpticas según la clasificación de restricción de crecimiento intrauterino atendidos en el Hospital Regional de Zacapa en el período del año 2013 al año 2017.

\section{Clasificación de restricción del crecimiento intrauterino en hijos de madres preeclámpticas}

El 75\% (54 casos) de recién nacidos presentaron una restricción de crecimiento intrauterino tipo II, mientras que un $25 \%$ (18 casos) presentaron restricción de crecimiento intrauterino tipo I.

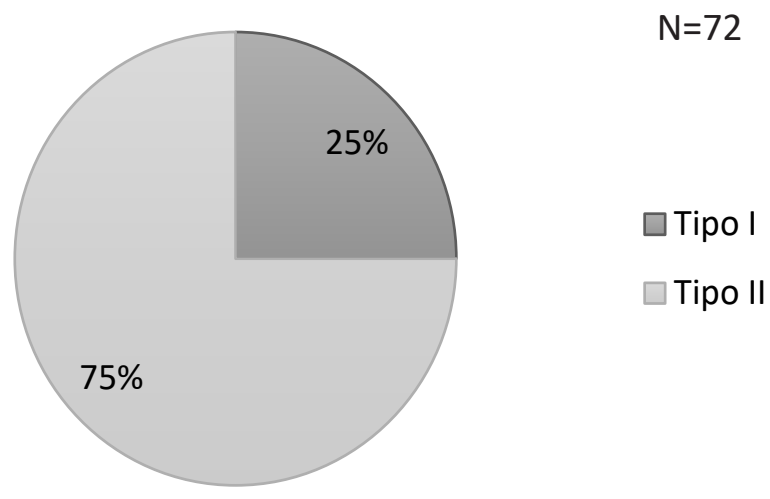

Figura 8. Distribución de pacientes hijos de madres preeclámpticas según la clasificación de restricción de crecimiento intrauterino atendidos en el Hospital Regional de Zacapa en el período del año 2013 al año 2017. 
Causa de ingreso a unidad de cuidados intensivos pediátricos en hijos de madres preeclámpticas

La principal causa de ingreso a unidad de cuidados intensivos pediátricos de los recién nacidos fue SDR (síndrome de dificultad respiratoria) con 86\% (12 casos), seguido de SAM (síndrome de aspiración meconial) con $14 \%$ ( 2 casos).

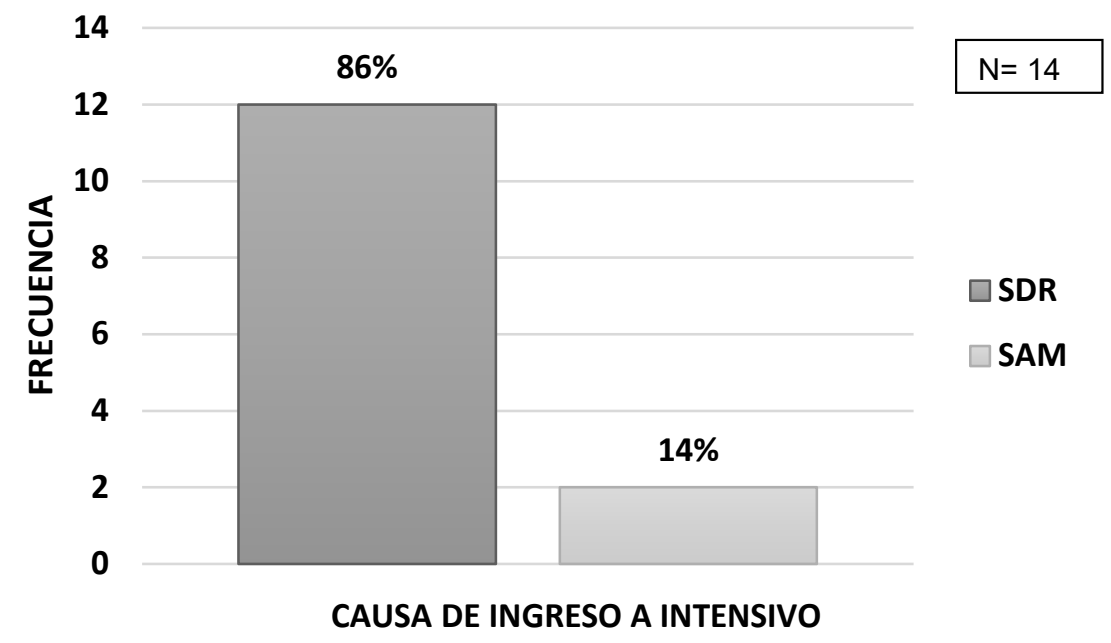

Figura 9. Distribución de pacientes hijos de madres preeclámpticas según causa de ingreso a unidad de cuidados intensivos pediátricos atendidos en el Hospital Regional de Zacapa en el período del año 2013 al año 2017.

\section{Causa de muerte en hijos de madres preeclámpticas}

La principal causa de muerte en los hijos de madres preeclámpticas fue óbito fetal con 59\% (10 casos), seguido de EMH (enfermedad de membrana hialina) con $24 \%$ (4 casos), luego choque séptico con $12 \%$ ( 2 casos) y por último SAM (síndrome de aspiración meconial) con 6\% (1 caso).

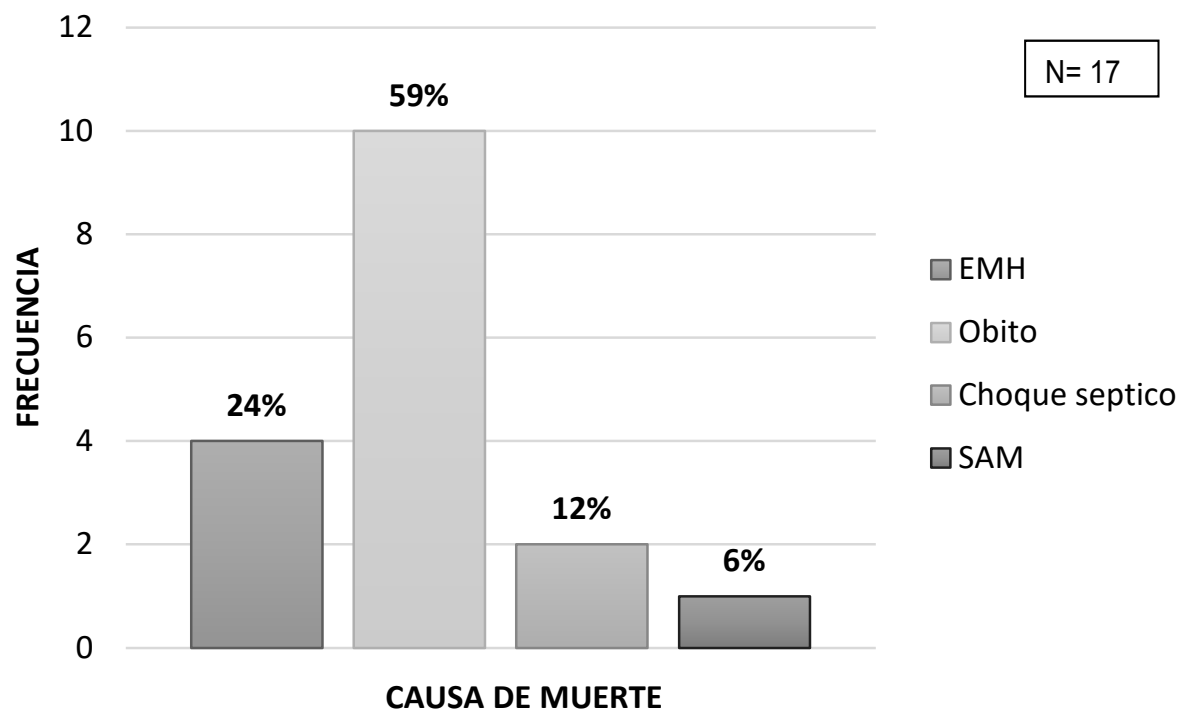

Figura 10. Distribución de pacientes hijos de madres preeclámpticas según causa de muerte atendidos en el Hospital Regional de Zacapa en el período del año 2013 al año 2017. 


\section{Discusión}

Los trastornos hipertensivos durante el embarazo constituyen una causa importante de morbi-mortalidad materna. En este estudio un 56\% de las pacientes preeclámpticas no presentaron ninguna complicación, mientras que un $44 \%$ si, siendo las complicaciones maternas más frecuentes el parto pretérmino $37 \%$, síndrome de Hellp 30\%, eclampsia $10 \%$ y oligohidramnios $10 \%$, y un $1 \%$ ( 1 caso) de insuficiencia renal. A diferencia de un estudio realizado en Ecuador por Caiza (2010:114) en el cual las complicaciones más frecuentes fueron síndrome de Hellp $71 \%$, eclampsia $18 \%$, insuficiencia renal $3 \%$ y preeclampsia refractaria $1 \%$, en donde no se muestra ningún caso de parto pretérmino ni de oligohidramnios.

En las pacientes con hipertensión durante el embarazo hay un aumento de la incidencia de eclampsia, desprendimiento de placenta, parto pretérmino, coagulación intravascular diseminada, hemorragia, insuficiencia renal, edema de pulmón, accidente cerebrovascular y muerte. En contraste con el presente estudio ya que no se presentó ningún caso de desprendimiento de placenta, coagulación intravascular diseminada, accidente cerebrovascular y muerte (Reece y Hobbins, 2010: 696). La (OMS, 2014) indica que en América Latina, una cuarta parte de las muertes maternas se relacionan con esas complicaciones, en el presente estudio no se reportó ninguna muerte materna, esto pudo ser causado por los criterios de exclusión tomados en cuenta para su realización.

La mayoría de recién nacidos hijos de madres preeclámpticas presentaron una edad gestacional al momento del nacimiento a término (37-42 semanas) $76 \%$ y un peso para edad gestacional adecuado $57 \%$, sin embargo un $61 \%$ de la población total presento algún tipo de complicación, siendo las más frecuentes BPN 21.5\%, PEG 20.6\% y RCIU 20.3\%. Concordando con el estudio nacional realizado por (López 2011: 80) en el cual llegó a la conclusión de que existe relación entre factores de riesgo maternos y restricción de crecimiento intrauterino o bajo peso al nacer, preeclampsia aumenta 12 veces este riesgo. En la Habana, Cuba, también se realizó un estudio por (García y colaboradores, 2012: 467-477), en el cual sus resultados muestran que los recién nacidos con peso inferior a 2,500 gramos se presentaron con mayor frecuencia en las pacientes con diagnóstico de preeclampsia, coincidiendo con el presente estudio en el cual BPN fue la complicación más frecuente en los recién nacidos de madres preeclámpticas.

Según (Dávila, 2016: 65-69), dentro de los hallazgos encontrados en los hijos de madres preeclámpticas existe evidencia de retardo de crecimiento intrauterino pequeño para edad gestacional, prematuridad, trastornos metabólicos, como hipoglicemia o hiperglicemia, hipocalcemia e hipopiridoxinemia, trastornos de coagulación, plaquetopenia, tiempo de tromboplastina parcial prolongado. Además hemorragia intraventricular, hiperbilirrubinemia, dificultad respiratoria (taquipnea transitoria, displasia broncopulmonar), persistencia de conducto arterioso, hipotensión, retinopatía, gastritis erosiva, predisposición a cuadros infecciosos, quimiotaxis y citoquinas, población de células de defensa así como CD34+ disminuidas, se asocia neutropenia y esto a su vez predispone a infección por hongos en particular cándida.

A diferencia del presente estudio en el cuál si se presentó PEG 20.6\%, RCIU 20.3\% y prematurez $12.9 \%$, trastornos metabólicos solamente hipoglicemia en $0.6 \%$, ictericia $0.3 \%$, SDR $4.3 \%$, cuadros infecciosos (choque séptico $0.6 \%$ y neumonía neonatal $0.6 \%$ ). 


\section{Agradecimientos}

Este trabajo ha sido posible gracias al apoyo y asesoría de las autoridades de la carrera médico y cirujano del Centro Universitario de Oriente y al apoyo del Hospital Regional de Zacapa.

\section{Referencias bibliográficas}

Caiza, S. (2010). Complicaciones materno-fetales asociadas a la preeclampsia atendidos en el hospital José María Velasco Ibarra-Tena en el periodo enero 2009 - enero 2010 (Tesis de Licenciatura) Riobamba, Ecuador, Escuela Superior Politécnica de Chimborazo, Facultad de Salud Pública. p.114. Recuperado de http://dspace.espoch.edu.ec/bitstream/ 123456789/1901/1/94T00080.pdf

Cunningham, F., Leveno, K., Bloom, S., Hauth, J., Spong, C., Dashe, J., Hoffman, B., Casey, B \& Sheffield, J. (2015). Williams obstetricia. 24 ed. p. 728-730 México: McGraw-Hill.

Dávila, C. (2016). Neonato de madre con preeclampsia: riesgo para toda la vida. Revista Peruana de Investigación Materno Perinatal 5 (1): 65-69. Recuperado de http://docplayer.es/72194827-Neonatode-madre-con-preeclampsia-riesgo-para-toda-la-vida.html. https://doi.org/10.33421/inmp.201657

García, R., Llera, A., Pachecho, A., Delgado, M., \& González, A. (2012). Resultados maternos perinatales de pacientes con preeclampsia. Revista Cubana de Obstetricia y Ginecología 38 (4): 467-477. Recuperado de Disponible en http://scielo.sld.cu/scielo. php?script=sci_arttext\&pid=S0138-600X2012000400004

López, C. (2011). Recién nacidos con restricción del crecimiento intrauterino (Tesis de Licenciatura) Universidad de San Carlos de Guatemala, Facultad de Ciencias Médicas, Guatemala. P. 80. Recuperado de http://biblioteca.usac.edu.gt/ tesis/05/05_8796.pdf

Organización Mundial de la salud -OMS. (2014). Recomendaciones de la OMS para la prevención y el tratamiento de la preeclampsia y la eclampsia. Recuperado de http://apps.who.int/iris/bitstream/10665/138405/1/9789243548333_spa.pdf

Preeclampsia Foundation. (2014). Mortalidad materna internacional y preclampsia: la carda mundial de la enfermedad. Recuperado de https://www.preeclampsia.org/es/informacion-de-salud/149-advocacy-awareness/332preeclampsia-and-maternal-mortality-a-global burden

Reece, E. \& Hobbins, J. (2010). Obstetricia clínica. 3 ed. Buenos Aires, Argentina: Editorial Médica Panamericana S.A. p 696. Recuperado de https://books.google.com.gt/books?id=RS11QMxGgA8C\&pg=PA684\&dq=PREECLAMPSIA\&hl=es419\&sa=X\&ved=0ahUKEwiJwZHEpo_ZAhWKt1kKHU4OB5UQ6AEIQjAE\#v=onepage \&q=PREECLAMPSIA\&f=false 


\section{Paola Tatiana Mariscal Chacón}

Médico y Cirujano, egresada del Centro Universitario de Oriente CUNORI- USAC. Actualmente es residente 1 de la Maestría de Ginecología y obstetricia en el Hospital Regional de Zacapa, Guatemala.

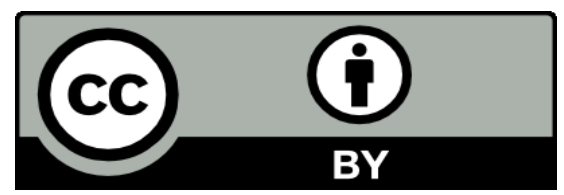

Este texto está protegido por una licencia CreativeCommons 4.0.

Usted es libre para compartir, copiar y redistribuir el material en cualquier medio o formato y adaptar el documento, remezclar, transformar y crear a partir del material para cualquier propósito, incluso comercialmente, siempre que cumpla la condición de atribución: usted debe reconocer el crédito de una obra de manera adecuada, proporcionar un enlace a la licencia, e indicar si se han realizado cambios. Puede hacerlo en cualquier forma razonable, pero no de forma tal que sugiera que tiene el apoyo del licenciante o lo recibe por el uso que hace. 